\title{
"Es gente fazil de moverse de una parte a otra". Diversidad en las estrategias de subsistencia y movilidad prehispánicas tardías (Sierras de Córdoba, Argentina)
}

\section{"Is people easy to move somewhere else": Diversity in the late pre-hispanic subsistence and mobility strategies (Sierras of Cordoba, Argentina)}

\author{
Matías E. Medina, Sebastián Pastor, Eduardo E. Berberián \\ CONICET - Área de Arqueología y Etnohistoria, Centro de Estudios Históricos "Prof. Carlos S.A. Segreti", \\ Miguel C. del Corro 308, Córdoba (5000), Argentina, \\ paleomedina@yahoo.com.ar
}

Recibido: 09-12-2013

Aceptado: 22-01-2014

\begin{abstract}
RESUMEN
El estudio arqueológico del Periodo Prehispánico Tardio de las Sierras de Córdoba (ca. 1100-360 AP, Argentina) durante décadas asumió que el desarrollo de prácticas agrícolas rápidamente derivó en una mayor dependencia de los cultivos -principalmente maíz (Zea mays) - y la sedentarización en poblados permanentes conformados por viviendas semi-enterradas o casas pozo. La información actual pone en duda este supuesto al sugerir que las poblaciones tardias desarrollaron patrones de subsistencia y movilidad flexibles, donde se alternaron las estrategias de acuerdo a la disponibilidad estacional de recursos silvestres. De este modo, los productos agrícolas sólo fueron un componente de una economía mixta en la que plantas y animales silvestres eran explotados en forma intensiva. El desarrollo de una estrategia de subsistencia diversificada se acompañaba de una alta movilidad residencial, momentos de dispersión/agregación de los grupos co-residentes y procesos estacionales de interrupción de las prácticas agrícolas para abastecerse de recursos de caza y recolección. El modelo es un ejemplo arqueológico en donde la incorporación de cultivos dio lugar a un patrón de subsistencia flexible sin derivar en una economía plenamente agrícola, con potencial comparativo para el estudio de la transición hacia la producción de alimentos en otras regiones del mundo.
\end{abstract}

Palabras clave: Período Prehispánico Tardio, Sierras de Córdoba (Argentina), Arqueología, Agricultura, Subsistencia, Movilidad.

\begin{abstract}
The archaeological study of Sierras of Córdoba Late Pre-Hispanic Period (1100-360 BP, Argentina) assumed during decades that the development of agriculture led to the sudden dependence of crops-mainly maize (Zea mays) - and to the sedentary way of life in pit-house villages. The current information questions this assumption by suggesting that the late pre-Hispanic peoples showed flexible subsistence and mobility patterns where the strategies were switched according to seasonal wild resource availability. Thus, farming was one component in a mixed foraging and cultivation economy where wild animals and plants were intensively exploited. The wide economic niche was accompanied by high residential mobility, co-residential group fission-fusion mechanism and the seasonal switch from farming to foraging wild resources. The model presents an archaeological example where the introduction of crops was followed by a flexible subsistence pattern and not by a full-time farming economy, which serves as a potential comparison to other cases in the world during the food producing transition.
\end{abstract}

Key words: Late Pre-Hispanic Period, Sierras of Córdoba (Argentina), Archaeology, Farming, Subsistence, Mobility. 


\section{Introducción}

Durante décadas, el estudio arqueológico del Periodo Prehispánico Tardío de las Sierras de Córdoba (Argentina; ca. 1100-360 AP) asoció la introducción de prácticas agrícolas con cambios culturales abruptos similares a los observados en otras partes del mundo. Se asumió que el desarrollo de cultivos rápidamente derivó en importantes innovaciones tecnológicas y organizativas, entre ellas una mayor dependencia de los cultivos - principalmente maíz (Zea mays) - y la sedentarización en poblados permanentes conformados por viviendas semi-enterradas o casas pozo (Aparicio 1939; Berberián 1984, 1999; Canals Frau 1953; González 1943; Laguens y Bonnín 2009; Outes 1911; Serrano 1945).

El interés en la introducción de la agricultura y su impacto sobre las estrategias de movilidad y subsistencia se ha incrementado en los últimos años. La incorporación de marcos de investigación regionales, conceptos de la Ecología del Comportamiento, nuevas técnicas de datación como el AMS y el desarrollo de análisis micro-botánicos (polen, fitolitos y gránulos de almidón) ha producido avances significativos. En este contexto, los sitios residenciales-agrícolas a cielo abierto, tradicionalmente considerados asentamientos sedentarios donde se realizaron actividades múltiples, son ahora sometidos a nuevas preguntas relacionadas con su dinámica de ocupación, estructuración espacial interna, cronología, características de la arquitectura doméstica y articulación con los espacios productivos, así como el lugar que ocuparon en el marco de los procesos sociopolíticos y de intensificación económica observados en la región.

El objetivo de este trabajo es presentar una breve discusión de estos planteamientos junto con el análisis de nuevos resultados, con especial énfasis en la diversidad de las estrategias de subsistencia y el patrón de movilidad practicado por las sociedades que habitaron las Sierras de Córdoba a fines del Holoceno.

\section{Agricultura y movilidad: evidencias para su discusión}

Hasta hace pocos años se entendió que la agricultura fue la principal estrategia económica sobre la que se organizó el resto de las actividades de subsistencia, la tecnología y la movilidad en el Período Prehispánico Tardío, asumiendo que los sitios con casas-pozo fueron el locus de poblaciones sedentarias. Dentro de esta propuesta, Berberián y Roldán (2003) establecieron un modelo que sostiene que las sociedades tardías del valle de Punilla desarrollaron una fuerte base agrícola hacia $1000 \mathrm{AP}$, con el establecimiento de sus sitios residenciales en el valle y la dispersión de chacras de cultivo en los alrededores. Parte de la población permanecía en forma estable en los sectores serranos bajos, donde se realizaban tareas domésticas, de recolección y fundamentalmente agrícolas. Las pampas de altura, distantes entre 15 y $20 \mathrm{~km}$, eran utilizadas transitoriamente para la captura de guanacos (Lama guanicoe) y venados de las pampas (Ozotoceros bezoarticus), en el marco de un sistema de alta movilidad logística dirigido a diversificar la subsistencia y minimizar el riesgo agrícola (Berberián y Roldán 2001).

Por su parte Laguens y Bonnín (2009) concluyen que la incorporación de la agricultura estuvo íntimamente asociada al desarrollo de una forma de vida sedentaria, aunque extienden su antigüedad hasta $c a$. 2000 AP. De acuerdo con estos autores, el sistema de asentamiento se extendió, aumentó en complejidad y se hizo permanente. Los recursos disponibles en los alrededores de los sitios -cultivos y algarrobales de Prosopis spp.- fueron explotados intensivamente. Laguens (1999) incluso estimó, atendiendo a la extensión de tierras cultivables en combinación con datos sobre la productividad del maíz (Zea mays) y la alubia (Phaseolus spp.), el aporte relativo de la agricultura a la dieta, que habría cubierto el $51,6 \%$ de las necesidades básicas.

Sin embargo, en todos los casos la importancia otorgada a la agricultura se apoyó en líneas de evidencias indirectas, como la ubicación de los sitios en terrenos potencialmente cultivables, su asociación con posibles instrumentos agrícolas (i.e. azuelas) y de molienda, estimación de la capacidad de carga, así como por la presencia de pequeños sitios a cielo abierto interpretados como campos de cultivos dispersos (Berberián y Roldán 2001; Laguens 1999; Medina y Pastor 2006). Excepcionalmente, la idea fue reforzada por estudios paleopatológicos sobre restos humanos, que señalan ambiguamente dietas ricas en hidratos de carbono (Bordach et al. 1991).

La limitada visibilidad arqueológica de la agricultura prehispánica favoreció durante años la sobrevaloración de los documentos coloniales de los siglos XVI-XVII en los estudios sobre el período tardío, con la definición de un sistema agrícola basado en el cultivo de maíz, alubias (Phaseolus sp.), calabaza (Cucurbita sp.), quinua (Chenopodium sp.) y tubérculos (Berberián 1987; Piana de Cuestas 1992; Serrano 1945). Por ejemplo, Berberián (1984) se apoyó en la descripción del cronista Diego Fernández para suponer la existencia de culti- 


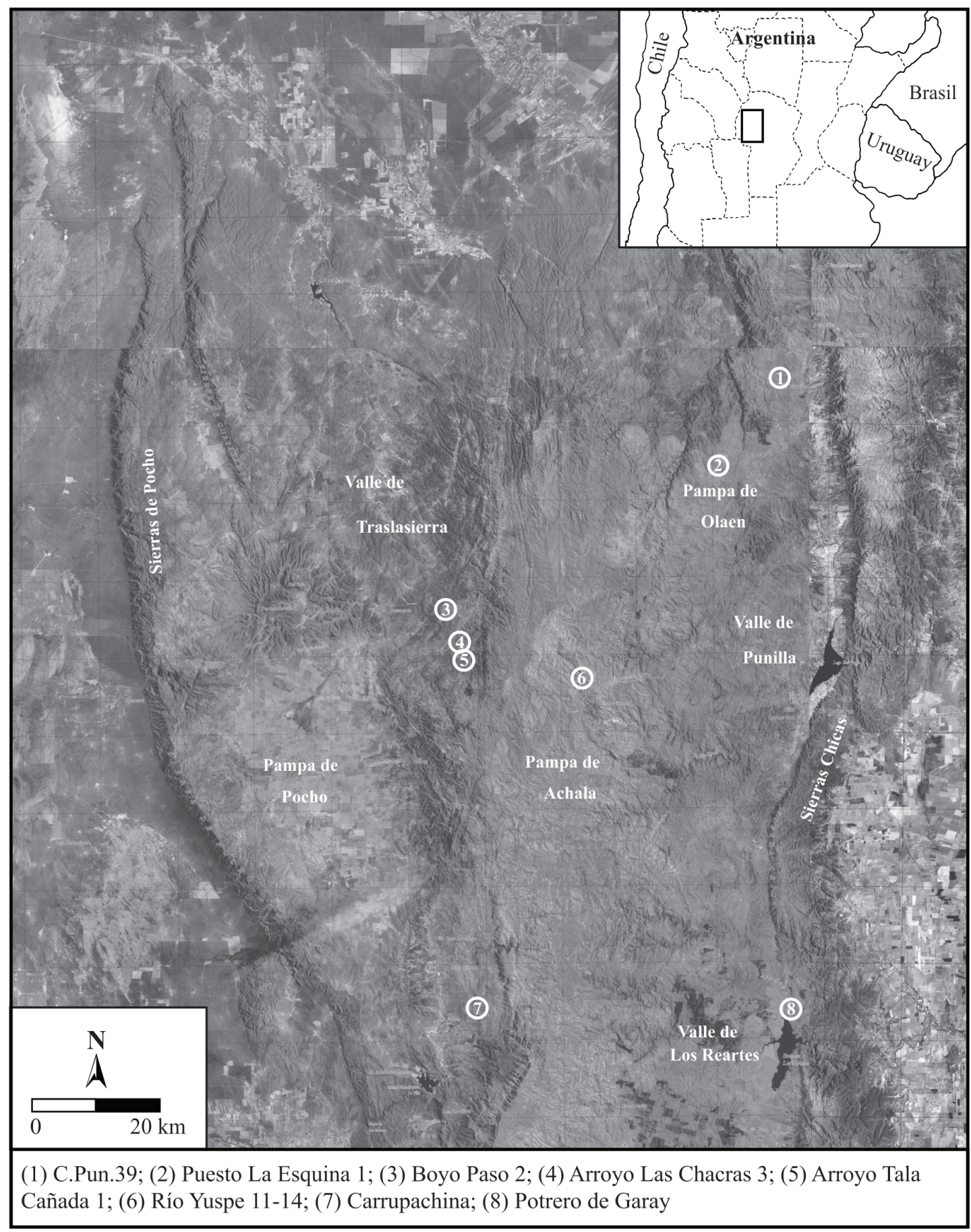

Figura 1.- Ubicación geográfica de los sitios arqueológicos mencionados en el trabajo.

vos en el entorno inmediato de Potrero de Garay (Fig. 1, Tabla 1), quien señala que los indígenas de Córdoba vivían en

...cuevas debajo de tierra, de suerte que, aunque lleguen a los pueblos, no se parecen sino por los maizales... (Berberián 1987: 53).
En las recientes excavaciones de los sitios residenciales a cielo abierto C.Pun.39, Puesto La Esquina 1, Boyo Paso 2 y Arroyo Tala Cañada 1 se recuperaron las primeras evidencias arqueológicas de cultígenos como el maíz (Zea mays), calabaza (Cucurbita sp.), alubia (Phaseolus vulga- 


\begin{tabular}{|l|l|l|l|l|}
\hline \multicolumn{1}{|c|}{ Sitio } & \multicolumn{1}{c|}{ Localización } & \multicolumn{1}{c|}{ Cronología } & $\begin{array}{c}\text { Test t de Ward } \\
\text { y Wilson }\end{array}$ & \multicolumn{1}{c|}{ Referencia } \\
\hline Potrero Garay & Valle de Los Reartes & $310 \pm 75$ AP (I-11-697) & ---- & Berberián (1984) \\
\hline C.Pun.39 & Valle de Punilla & $\begin{array}{l}854 \pm 39 \text { AP (AA2338) } \\
716 \pm 39 \text { AP (AA2339) } \\
525 \pm 37 \text { AP (AA64819) }\end{array}$ & Diferentes & Medina (2010) \\
\hline $\begin{array}{l}\text { Puesto La } \\
\text { Esquina 1 }\end{array}$ & Pampa de Olaen & $\begin{array}{l}365 \pm 38 \text { AP (AA64816) } \\
362 \pm 43 \text { AP (AA64815) }\end{array}$ & Indistinguibles & Medina (2010) \\
\hline Boyo Paso 2 & Valle de Salsacate & $750 \pm 70$ AP (LP-2932) & --- & Este trabajo \\
\hline $\begin{array}{l}\text { Arroyo Tala } \\
\text { Cañada 1 }\end{array}$ & Valle de Salsacate & $\begin{array}{l}1028 \pm 40 \text { AP (AA64820) } \\
900 \pm 70 \text { AP (LP-1511) }\end{array}$ & Indistinguibles & $\begin{array}{l}\text { Pastor y Berberián } \\
(2007)\end{array}$ \\
\hline $\begin{array}{l}\text { Arroyo Las } \\
\text { Chacras 3 }\end{array}$ & Valle de Salsacate & $917 \pm 37$ AP (AA100670) & --- & Este trabajo \\
\hline
\end{tabular}

Tabla 1.- Dataciones radiocarbónicas y Test $\mathrm{t}$ de Ward y Wilson (1978) de los sitios arqueológicos mencionados en este trabajo. Nota: El Test t de Ward y Wilson (1978) evalúa estadísticamente la posibilidad de que las dataciones tengan o no la misma edad verdadera.

ris) y haba de lima (P. lunatus), tanto en forma de macro-restos carbonizados como de micro-fósiles asociados a sedimentos, instrumentos de molienda y tiestos cerámicos (Fig. 1; Tabla 1 y 2) (Medina y López 2007; Medina et al. 2009; Pastor y López 2010). Las frecuencias excepcionales de polen de Chenopodiaceae-Amaranthaceae en sedimentos de C.Pun.39 datados en $c a$. 500 AP es indicativa de que plantas similares a cultivos andinos como la quinua (Chenopodium spp.) y amaranto (Amaranthus spp.) crecían abundantemente en los alrededores del sitio, probablemente en parcelas agrícolas (Medina et al. 2008). La identificación en Arroyo Tala Cañada 1 de un rasgo de surcos aproximadamente paralelos, similares a los generados mediante el laboreo de la tierra con azadas, junto con micro-fósiles de partes no comestibles de Zea mays y Phaseolus sp. en los sedimentos, corroboran el cultivo in situ a escasos metros de las viviendas (Pastor y Berberián 2007). Dos cotiledones de $P$. vulgaris provenientes de Arroyo Tala Cañada 1 y C.Pun.39 fueron datados en forma directa por AMS, con valores de $1028 \pm 40$ AP y $525 \pm 36$ AP que confirman la presencia prehispánica de este cultígeno (Medina et al. 2009; Pastor 2007a).

Sin embargo, es imposible sostener que la agricultura haya sido la estrategia económica de mayor jerarquía a partir de los restos arqueobotánicos. Menos aún como para justificar una estrategia de movilidad con alto grado de sedentarismo. En lo que hace específicamente a los macro-restos, el número de especímenes recuperados es relativamente bajo y sólo se han conservado en estado carbonizado, lo cual indica malas condiciones de preservación (Tabla 2). Aun cuando las técnicas de recuperación han mejorado con el empleo de flotación y zarandas de mallas finas para cernir los sedimentos (López 2005), la discusión continúa basándose en escasos fragmentos de $P$. vulgaris, P. lunatus y Zea mays (Tabla 2), que impiden el empleo de la cuantificación como medio para interpretar la importancia relativa de los cultivos. A esto se suma que sólo dos especímenes de $P$. vulgaris fueron datados en forma directa, un aspecto fundamental para discutir la asociación del cultígeno y la cronología radiocarbónica.

El estudio de micro-fósiles en sedimentos, artefactos de molienda y fragmentos cerámicos ha permitido identificar una mayor riqueza de plantas cultivadas aún bajo estas condiciones, incluso de especies de difícil preservación en el registro arqueológico como Chenopodium o Amaranthus (Medina et al. 2008; Pastor y López 2010). Sin embargo, los resultados también presentan problemas para realizar estimaciones de su importancia en la dieta prehispánica y justificar una estrategia de movilidad sedentaria, dado que la probabilidad de recuperar polen, gránulos de almidón y/o fitolitos de un cultígeno depende de la capacidad de la planta de producir polen, de acumular energía en forma de almidón y/o de absorber sílice en sus células. Por ello, las frecuencias taxonómicas relativas deben ser tomadas con cautela, sin descartar el posible consumo de otras plantas domesticadas o silvestres con baja o nula producción de polen, almidón o fitolitos (Pearsall 2004). 


\begin{tabular}{|c|c|c|c|c|}
\hline Sitio & Familia & Identificación & $\begin{array}{l}\text { Nombre } \\
\text { común }\end{array}$ & Observaciones \\
\hline C.Pun.39 & Poaceae & Zea mays & Maíz & $\begin{array}{l}1 \text { cariopsis carbonizada; Fitolitos } \\
\text { del morfotipoWavy/Ruffle top } \\
\text { rondel adheridos a tiestos cerámicos } \\
\text { e instrumentos de molienda }\end{array}$ \\
\hline C.Pun.39 & Fabaceae & $\begin{array}{l}\text { Phaseolus vulgaris aff. } \\
\text { var. vulgaris }\end{array}$ & Alubia & $\begin{array}{l}8 \text { fragmentos de cotiledones } \\
\text { carbonizados }\end{array}$ \\
\hline C.Pun.39 & Fabaceae & Phaseolus cf. P. lunatus & $\begin{array}{l}\text { Haba de } \\
\text { lima }\end{array}$ & $\begin{array}{l}4 \text { fragmentos de cotiledones } \\
\text { carbonizados }\end{array}$ \\
\hline C.Pun.39 & Cucurbitaceae & Cucurbita sp. & $\begin{array}{l}\text { Calabaza, } \\
\text { cayote, etc. }\end{array}$ & $\begin{array}{l}\text { Fitolitos del morfotipo sphera } \\
\text { facetate adheridos a tiestos } \\
\text { cerámicos }\end{array}$ \\
\hline C.Pun.39 & $\begin{array}{l}\text { Chenopodiaceae- } \\
\text { Amaranthaceae }\end{array}$ & $\begin{array}{l}\text { Chenopodiaceae- } \\
\text { Amaranthaceae }\end{array}$ & $\begin{array}{l}\text { Quinua- } \\
\text { Amaranto? }\end{array}$ & $\begin{array}{l}\text { Polen en sedimentos datados en } \\
525 \pm 36 \text { AP }\end{array}$ \\
\hline C.Pun.39 & $\begin{array}{l}\text { Chenopodiaceae- } \\
\text { Amaranthaceae }\end{array}$ & $\begin{array}{l}\text { Chenopodium sp.- } \\
\text { Amaranthus sp. }\end{array}$ & $\begin{array}{l}\text { Quinua- } \\
\text { Amaranto? }\end{array}$ & $\begin{array}{l}\text { Gránulos de almidón adheridos a } \\
\text { tiestos cerámicos }\end{array}$ \\
\hline $\begin{array}{l}\text { Puesto La } \\
\text { Esquina } 1\end{array}$ & Poaceae & Zea mays & Maíz & $\begin{array}{l}1 \text { fragmento de marlo carbonizado; } \\
\text { Fitolitos del morfotipo Wavy/ } \\
\text { Ruffle top rondel adheridos a tiestos } \\
\text { cerámicos }\end{array}$ \\
\hline $\begin{array}{l}\text { Puesto La } \\
\text { Esquina } 1\end{array}$ & Fabaceae & $\begin{array}{l}\text { Phaseolus vulgaris aff. } \\
\text { var. vulgaris }\end{array}$ & Alubia & $\begin{array}{l}2 \text { fragmentos de cotiledones } \\
\text { carbonizados }\end{array}$ \\
\hline $\begin{array}{l}\text { Puesto La } \\
\text { Esquina } 1\end{array}$ & Fabaceae & Phaseolus cf. P. lunatus & $\begin{array}{l}\text { Haba de } \\
\text { lima }\end{array}$ & $\begin{array}{l}4 \text { fragmentos de cotiledones } \\
\text { carbonizados }\end{array}$ \\
\hline Boyo Paso 2 & Poaceae & Zea mays & Maíz & Fragmento de cúpula carbonizada \\
\hline $\begin{array}{l}\text { Arroyo Tala } \\
\text { Cañada } 1\end{array}$ & Fabaceae & $\begin{array}{l}\text { Phaseolus vulgaris aff. } \\
\text { var. vulgaris }\end{array}$ & Alubia & $\begin{array}{l}13 \text { fragmentos de cotiledones } \\
\text { carbonizados }\end{array}$ \\
\hline $\begin{array}{l}\text { Arroyo Tala } \\
\text { Cañada } 1\end{array}$ & Fabaceae & Phaseolus cf. P. lunatus & $\begin{array}{l}\text { Haba de } \\
\text { lima }\end{array}$ & $\begin{array}{l}5 \text { fragmentos de cotiledones } \\
\text { carbonizados }\end{array}$ \\
\hline $\begin{array}{l}\text { Arroyo Tala } \\
\text { Cañada } 1\end{array}$ & Fabaceae & Phaseolus sp. & Alubia & $\begin{array}{l}\text { Fitolitos del morfotipo unciform } \\
\text { unicelular haircell en sedimentos }\end{array}$ \\
\hline $\begin{array}{l}\text { Arroyo Tala } \\
\text { Cañada } 1\end{array}$ & Poaceae & Zea mays & maíz & $\begin{array}{l}\text { Fitolitos del morfotipo cross-shaped } \\
\text { en sedimentos }\end{array}$ \\
\hline $\begin{array}{l}\text { Arroyo Tala } \\
\text { Cañada } 1\end{array}$ & Cucurbitaceae & Cucurbita sp. & $\begin{array}{l}\text { Calabaza, } \\
\text { cayote, etc. }\end{array}$ & $\begin{array}{l}\text { Fitolitos del morfotipo sphera } \\
\text { facetate en sedimentos }\end{array}$ \\
\hline
\end{tabular}

Tabla 2.- Plantas cultivadas recuperadas en sitios prehispánicos tardíos de las Sierras de Córdoba (Argentina).

La evaluación de la importancia de los cultígenos en la subsistencia prehispánica requiere, entonces, ir más allá de los restos arqueobotánicos y examinar el contexto general, como el patrón de asentamiento, el resto de las actividades de subsistencia y la organización de la tecnología (Chil- ton 1999), aspectos que serán en parte discutidos infra.

La información actualmente disponible también cuestiona el supuesto de que los asentamientos residenciales a cielo abierto fueran utilizados en forma permanente o sedentaria ${ }^{1}$. La primera línea 
de evidencia que sugiere el abandono estacional de los sitios residenciales se relaciona con la baja visibilidad arqueológica de las estructuras habitacionales. En este sentido, los datos sobre las características de la arquitectura de la vivienda son escasos en las Sierras de Córdoba. En los casos excepcionales en que se detectaron viviendas, éstas fueron construidas con materiales perecederos y con una baja inversión de energía (i.e. casas-pozo; Berberián 1984).

La estructura y organización interna de los sitios residenciales recibió un tratamiento concreto durante las extensas intervenciones estratigráficas en el sitio Potrero de Garay, donde se identificaron cuatro viviendas semi-subterráneas de planta rectangular, de unos $6 \mathrm{~m}$ de largo por $4.5 \mathrm{~m}$ de ancho (Figs 1 y 2a) (Berberián 1984). Las paredes estaban excavadas verticalmente sobre la matriz de sedimentos, sin ningún tipo de revoque ni columnas de piedra, ni tampoco evidencias de reparaciones o remodelaciones que sugieran una utilización a largo plazo. En su perímetro se ubicaron una serie de agujeros donde se colocaron los postes que sostenían el techo. El piso de los recintos era plano, consolidado pero de escaso espesor, sin preparación evidente más que el pisoteo de la actividad cotidiana. No se detectaron fogones formales en los pisos, aunque sí depresiones asociadas a tierra calcinada y carbón disperso (Berberián 1984). Debajo de los fondos de vivienda se encontraron tumbas con entierros.

Las investigaciones en Potrero de Garay permitieron confirmar y conocer con mayor detalle una serie de apreciaciones efectuadas previamente en otros asentamientos (González 1943; Serrano 1945), así como las descripciones contenidas en las fuentes coloniales tempranas. Como ejemplo de esto último, el cronista Pedro Cieza de León, en su relato de la entrada de Diego de Rojas al Tucumán (1543-1546), apuntaba que para la construcción de sus viviendas los indígenas de las Sierras de Córdoba

...cavaban la tierra hasta que ahondando en ella quedaban dos paredes, poniendo la madera armaban las casas, cobijándolas de paja a manera de chozas... (Berberián 1987: 130).

En la mayoría de los sitios, como los recientemente excavados C.Pun.39, Puesto La Esquina 1, Carrupachina, Boyo Paso 2, Arroyo Tala Cañada 1 y Arroyo Las Chacras 3, la presencia de recintos habitacionales sólo se infiere a partir de la detección de superficies consolidadas, fogones informales, agujeros de postes y materiales en planta, que se superponen y difícilmente conforman una estructura permanente que permita defender un concepto de estabilidad residencial (Fig. 2) (Pastor y Berberián 2007; Medina 2008; Medina et al. 2008; Pastor et al. 2013). Una situación similar ocurre con la habitual ausencia de basureros bien definidos, que impide sostener la existencia de un "sedentarismo" en base a evidencia negativa y conduce a plantear un alto grado de movilidad residencial entre los grupos que habitaron la región. En consecuencia, el registro arqueológico apoya la hipótesis de un uso estacional de los sitios residenciales a cielo abierto, con claras señales de flexibilidad en las prácticas de asentamiento y especialmente en cuanto a la arquitectura doméstica, de características efímeras, bajo costo y no planificada para un uso a largo plazo.

Las crónicas del tiempo de la conquista apoyan esta interpretación al señalar, en un claro contexto de pérdida de territorios y desestructuración social, el uso de estrategias de movilidad de raíz prehispánica ante situaciones de explotación y/o inestabilidad ambiental. Por ejemplo, Antonio Suárez Mexía declara en 1587-1589 que la población indígena de las sierras “....es gente fazil de moverse de una parte a otra..." (Piana de Cuestas 1992), mientras que un documento de 1609 menciona un caso en donde los indígenas "...desampararon el pueblo y con sus caciques y sus indios se fueron a hazer las chozas en los montes junto a sus chacaras..." (Bixio y Berberián 1984), indicando la flexibilidad para mudar y construir viviendas como respuesta a la escasez de alimentos o bien para evitar contacto con los españoles. Martín de Zurita (1983) también nota la falta de permanencia de las comunidades serranas:

...los indios de su repartimiento questan en la Sierra de Viarapa a la vertiente della los traerá en los términos que le es mandado pudiéndolos traer por quanto ahora están necesitados de comida y asi están fuera de sus pueblos... (AHPC Esc I, Leg I, Exp 5 15841585)

, marcando el profundo conocimiento de las poblaciones nativas para mover sus residencias y un claro uso diversificado del paisaje y sus recursos.

Los datos obtenidos en prospecciones, sondeos y excavaciones, junto con la indagación crítica de la información previa, sugieren diferencias en cuanto a la intensidad, duración y continuidad de uso de los distintos asentamientos (Medina 2008; Pastor 2007a; Pastor et al. 2013)². En este sentido, existe una clara diversidad en las estrategias de ocupación de los sitios, posiblemente relacionada con la alternancia estacional entre agricultura y caza-recolección, con rangos de variación que van desde la ocupación infrecuente y/o única a escala arqueológica hasta sitios con evidencias de un uso recurrente y depósitos profundos (Tabla 3). Las 


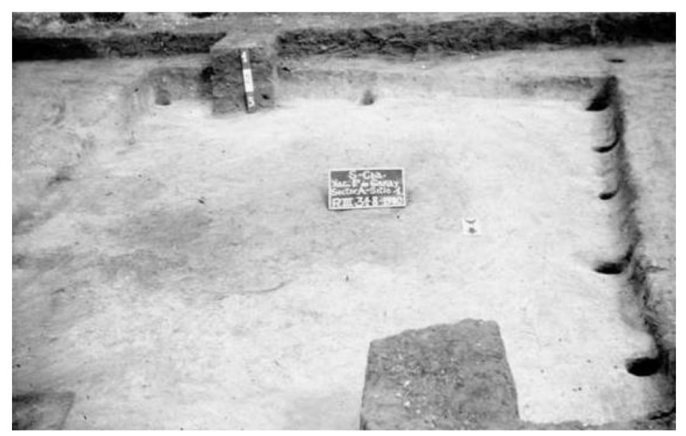

a)

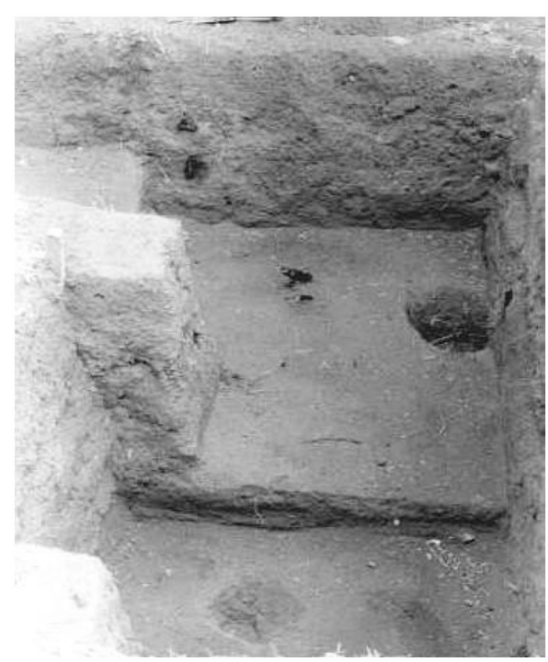

c)

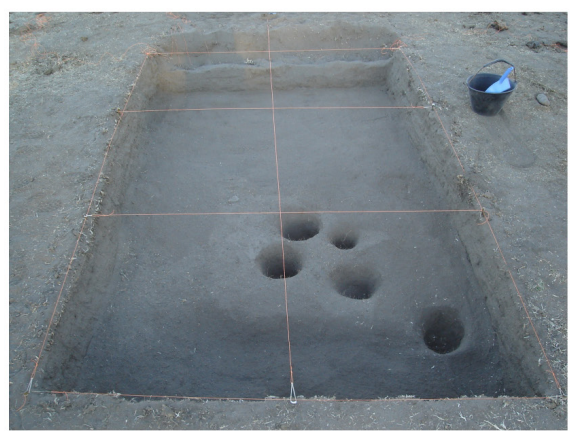

b)

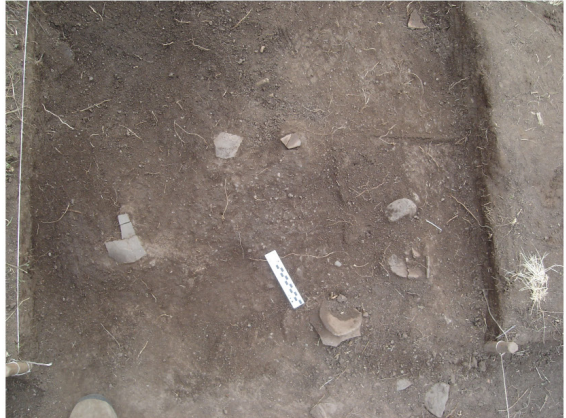

d)

Figura 2.- Casa-Pozo de Potrero de Garay; b. Piso de ocupación y agujeros de poste en Boyo Paso 2; c. Piso de ocupación y agujero de poste en Arroyo Tala Cañada 1; d. Materiales en planta en Arroyo Las Chacras 3.

\begin{tabular}{|c|c|c|}
\hline Categoría de sitio & Frecuencia de ocupación & Duración de la ocupación \\
\hline \multirow{5}{*}{ Sitio residencial a cielo abierto } & \multirow{5}{*}{$\begin{array}{l}\text {-única } \\
\text {-repetida }\end{array}$} & -muy largo plazo (>12 meses) \\
\hline & & -largo plazo (6-12 meses) \\
\hline & & -mediano plazo (2-6 meses) \\
\hline & & -corto plazo (8 semanas-2 meses) \\
\hline & & -muy corto plazo (1-14 días) \\
\hline \multirow{5}{*}{$\begin{array}{l}\text { Alero rocoso con ocupación tipo } \\
\text { doméstica }\end{array}$} & \multirow{5}{*}{$\begin{array}{l}\text {-única } \\
\text {-repetida }\end{array}$} & -muy largo plazo (>12 meses) \\
\hline & & -largo plazo (6-12 meses) \\
\hline & & -mediano plazo (2-6 meses) \\
\hline & & -corto plazo ( 8 semanas-2 meses) \\
\hline & & -muy corto plazo (1-14 días) \\
\hline
\end{tabular}

Tabla 3.- Modelo de las alternativas concernientes a la frecuencia y duración de las ocupaciones. El sombreado indica el máximo de probabilidad en el prehispánico tardío de las Sierras de Córdoba (Argentina). La duración en tiempo es especulativa y sólo se presenta para proveer de un marco de referencia de grano grueso a la discusión.. 
dataciones a partir de muestras obtenidas aproximadamente en columna en C.Pun.39, asociadas a reiteradas ocupaciones, y en Puesto La Esquina 1, Arroyo Las Chacras 3 y Arroyo Tala Cañada 1, que sugieren a escala arqueológica un único evento de ocupación, acompañan la hipótesis de variabilidad en las dinámicas ocupacionales (Tabla 1). Los valores excepcionales de tipos polínicos indicadores de disturbio antrópico continuo en sedimentos de C.Pun.39 son consistentes con procesos repetidos de abandono y reocupación, en la misma línea que sus dataciones radiocarbónicas (Medina et al. 2008).

Los indicadores faunísticos de estacionalidad (i.e. huesos de Tupinambis y cáscaras de huevos de Rheidae), junto con evidencias de actividades agrícolas (semillas, surcos, instrumentos agrícolas, etc.) y de recolección (Prosopis sp., Geoffroea decorticans, P. vulgaris var. aborigenus, Ziziphus mistol, etc.), sugieren que la ocupación y reocupación de los sitios coincidió con la primaveraverano, momento del año en que debía realizarse la siembra y/o estaban disponibles los recursos silvestres en el entorno inmediato (Medina 2008; Medina et al. 2007; Medina et al. 2009; Medina y Pastor 2012; Pastor y Berberián 2007). En la misma línea, el predominio del estadio 2 de Beherensmeyer (1978) en los perfiles de meteorización de los conjuntos faunísticos, con valores superiores al $60 \%$, se aleja de lo esperado para sitios con historias de ocupación continua y prolongada, donde los diferentes estadios tenderían a distribuirse con frecuencias homogéneas (Gifford et al. 1980). En cambio, aportan información para discutir que la mayoría de los especímenes se depositaron en el mismo rango de tiempo y que estuvieron expuestos durante lapsos significativos a los agentes atmosféricos (Medina y Pastor 2012). En consecuencia, la evidencia tafonómica sugiere que los sitios no fueron ocupados en forma continua como tradicionalmente se sostuvo, ya que pudieron existir hiatos estratigráficamente indiferenciables entre las distintas ocupaciones donde se produjo el lento enterramiento de los restos óseos.

El abandono estacional de los poblados tardíos también se apoya en la información proveniente del resto del paisaje arqueológico de las Sierras de Córdoba. El uso intensivo de ambientes con condiciones adversas para la producción agrícola pero ricos en recursos de caza-recolección (Tabla 3 ), como las pampas de altura y las Sierras Occidentales, da cuenta de fenómenos de dispersión estacional de los grupos co-residenciales (Pastor et al. 2012). En este sentido, los pastizales de altura sobre las Sierras Grandes cumplieron un rol fundamental en el proceso de dispersión invernal de los grupos que co-residían en poblados. Las excavaciones en los abrigos rocosos Río Yuspe 11 y 14, localizados en la porción nororiental de Pampa de Achala, informan que la ocupación de estos espacios se focalizó en la captura de ungulados silvestres de alto rendimiento económico - i.e. guanacos y venados de las pampas- (Pastor 2005, 2007b; Pastor y Medina 2005). En cambio, las ocupaciones tardías de Guasapampa y Serrezuela (Sierras Occidentales), con abundantes expresiones de arte rupestre, se vincularon con el aprovechamiento de la maduración relativamente temprana de los frutos chaqueños en los sectores de menor altitud (Pastor 2010; Recalde 2008-09).

La existencia de campos de cultivo dispersos y la ausencia de un registro arquitectónico vinculado a la producción (i.e. andenerías, canchones, muros de contención, acequias, etc.) son consistentes con una agricultura de temporal, con baja inversión en infraestructura y mínimos cuidados (Medina 2008; Pastor 2007a; Pastor y López 2010). Este tipo de práctica impone comúnmente la necesidad de cierta movilidad para contrarrestar el agotamiento de los suelos y la invasión de malezas, lo cual refuerza patrones pre-existentes de uso estacional y diversificado del paisaje. Además, la identificación de micro-fósiles y macro-restos de cultivos no permite sostener que recursos agrícolas como el maíz hayan sido tan centrales en la subsistencia como para justificar una estrategia sedentaria, aspecto discutido en otros sectores del texto.

La recuperación de abundantes artefactos pesados y difíciles de transportar, como percutores, morteros, molinos y manos de molinos, así como restos de pigmentos, nódulos, núcleos de materias primas no locales y vasijas grandes (Dantas y Figueroa 2008; Medina 2008, 2010; Pastor 2007a), sugiere conductas orientadas a aprovisionar lugares (Kuhn 1995) y no un sedentarismo estricto como tradicionalmente se interpretó. En este sentido, se trata de objetos explotados por debajo de su potencial que comúnmente se abandonan en los campamentos-base para ser reutilizados nuevamente a lo largo de las sucesivas reocupaciones (Eerkens 2003; Graham 1994; Stevenson 1982).

Los estudios zooarqueológicos, arqueobotánicos, bioarqueológicos y de organización de la tecnología apuntan hacia dietas mixtas donde los recursos silvestres continuaban siendo explotados en forma intensiva a través del uso diversificado y estacional del paisaje. Los datos isotópicos y patológicos de restos humanos, así como los restos arqueobotánicos de Prosopis sp., Z. mistol, P. vulgaris var. aborigenus, $G$. decorticans y el uso intensivo de ambientes no favorables para la agricultura, apoyan la hipótesis de que el maíz no fue 
central en la economía tardía (González y Fabra 2011; Laguens et al. 2009; Medina y López 2007; Medina et al. 2009, Pastor 2010; Recalde 2008, 2009). La incidencia de las actividades cinegéticas también está señalada por la abundancia de restos óseos de camélidos y cérvidos, así como una amplia gama de aves, reptiles y pequeños mamíferos, todos ellos con evidencias de procesamiento antrópico o de haber sido potencialmente consumidos (Medina y Pastor 2012; Medina et al. 2011). Los cambios operados en los sistemas de armas, ca. 1500-1100 AP., con la producción de distintos diseños de puntas de proyectil, empleo selectivo de rocas, incorporación de materias primas óseas y del arco como forma de propulsión, sugiere que la captura de presas silvestres no era un simple complemento de la dieta, sino una estrategia económica significativa e incluso más relevante que en momentos anteriores (Medina 2008).

La última evidencia arqueológica clave en esta discusión es la cerámica, cuya asociación directa con la agricultura y por extensión con el sedentarismo cabe poner en cuestión, apoyando la noción de un patrón de subsistencia diversificado donde una variedad de cultígenos, plantas y animales silvestres fueron el núcleo de la dieta. El análisis de los indicadores de formas sugiere que las ollas glo- bulares de paredes finas y bocas restringidas prevalecen en los conjuntos (Fig. 3) (Dantas y Figueroa 2008; Medina 2008, 2010). Se interpreta que su frecuencia responde al diseño versátil y transportable de estos artefactos (sensu Nelson 1991), que se anticipa a distintas situaciones de uso, siendo óptimos para cumplir con un amplio rango de tareas en un contexto de alta movilidad residencial. En este sentido, las ollas fueron contenedores aptos para cumplir con múltiples actividades, desde el almacenaje (fundamental para el consumo diferido y la viabilidad de las futuras cosechas) hasta la cocción de frutos, semillas, carne y grasa ósea, además del procesamiento de productos agrícolas que requieren largos períodos de hervido para ser aceptables para el consumo, como es el caso del maíz y la alubia (Arnold 1999; Skibo y Blinman 1999). Asimismo, facilitaron la elaboración de productos alternativos como bebidas alcohólicas, cuya fermentación puede llegar a romper otro tipo de contenedores (Skibo y Blinman 1999). Los estudios de huellas de uso (hollín, sustancias adheridas, desgaste, etc.), junto a la consideración de los diámetros de las bocas, presencia/ausencia de cuello y el acabado final de las superficies, corroboran la versatilidad y el amplio rango de contextos de uso de las morfologías globulares (Medina 2010).

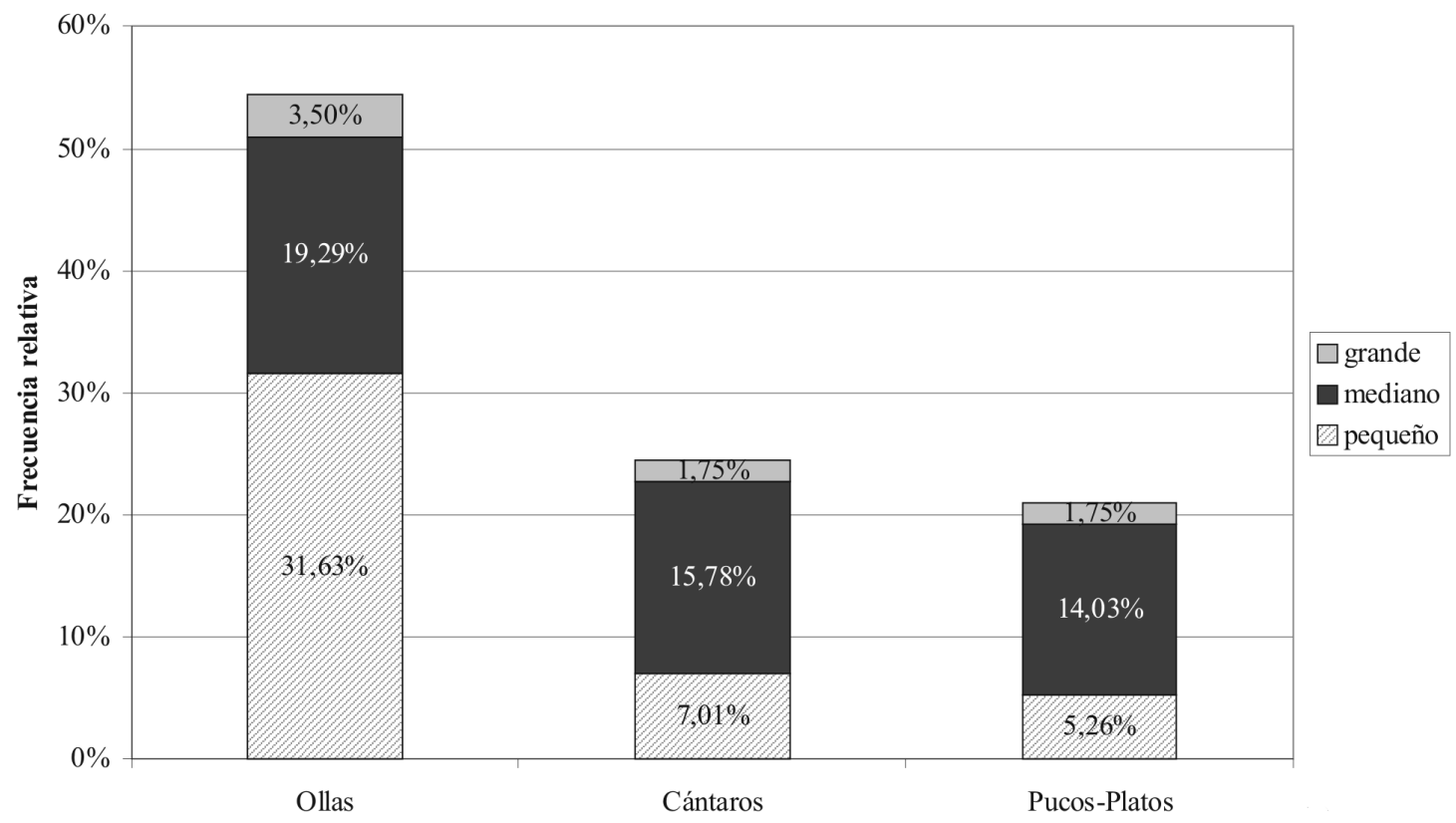

Formas generales

Figura 3.- Porcentaje de las formas generales y tamaños de los recipientes cerámicos reconstruidos en Puesto La Esquina $1(\mathrm{n}=57)$. Pucos y platos son integrados en una misma categoría inclusiva, por tratarse de recipientes empleados para el consumo de alimentos. 


\section{Discusión}

Durante décadas las investigaciones arqueológicas en las Sierras de Córdoba centraron su atención en diferenciar una etapa "precerámica" de una "agroalfarera", esquema que valoró las innovaciones tecnológicas y favoreció una concepción dicotómica y unidireccional entre ambos tipos de organización (Pastor 2007a). Hasta hace pocos años esta noción se mantuvo con leves modificaciones al definir el Período Prehispánico Tardío (ca. 1100-300 AP) por la presencia de "comunidades productoras de alimentos", "sociedades agrícolas a pequeña escala", "formativas" o con una "estrategia predominante agrícola", donde la caza y la recolección eran sólo actividades complementarias (Berberián 1999; Berberián y Roldán 2001, 2003; Medina y Pastor 2006).

A la luz de la información actual, el dualismo entre "caza-recolección" y "agricultura" se ha transformado en un obstáculo para el desarrollo de las investigaciones arqueológicas. En primer lugar, porque reduce la diversidad arqueológica de las Sierras de Córdoba en categorías artificiales de poca utilidad (Muscio 2004; Pastor 2007a). Por otra parte, al considerar estas estrategias como mutuamente excluyentes y no como prácticas en alternancia, el estudio de sus posibles combinaciones, oscilaciones o reversiones queda fuera de toda agenda de investigación (Diehl 2005; Layton et al. 1991). Finalmente, porque su uso genera numerosas preguntas y problemas de difícil resolución con el registro arqueológico, sobre todo en cuanto a los criterios necesarios para definir a una sociedad como "agrícola" (Smith 2001).

En un intento por salvar parte de esta situación, Winterhalder y Kennett (2006: 4) sostienen que el término "agricultores" debe utilizarse para poblaciones donde los recursos domesticados satisfacen aproximadamente un $75 \%$ de las necesidades básicas de subsistencia. Aun cuando esta definición es útil para el estudio de situaciones etnográficas, no tiene en cuenta que el carácter fragmentario del registro arqueológico determina serias limitaciones para estimar la incidencia relativa de cada actividad económica. Numerosos estudios sugieren, en cambio, que las economías prehistóricas que combinaban recursos domésticos y silvestres fueron comunes y muy estables en términos adaptativos (Smith 2001).

Las evidencias arqueológicas utilizadas para defender la existencia de cultivos prehispánicos en las Sierras de Córdoba demuestran que los cultígenos-especialmente el maíz- fueron importantes en términos económicos, pero no permiten sostener con firmeza que la agricultura haya sido la estrategia de mayor jerarquía o que las sociedades tardías dependieran de ella para su reproducción. Otras observaciones, en cambio, como la intensidad de uso de los ambientes serranos de altura y de espacios forestales que bordean la llanura, los restos arqueofaunísticos, la diversidad y abundancia de puntas de proyectil, los restos arqueobotánicos de frutos chaqueños, la abundancia de fragmentos de cáscaras de huevo en algunos sitios y las primeras tendencias isotópicas de restos humanos, sugieren que las prácticas extractivas no fueron simples actividades complementarias.

La adopción de cultígenos no parece haber marcado una frontera precisa entre "caza-recolección" y "agricultura". Al contrario, pone en evidencia que los límites entre ambas estrategias suelen ser difusos, en tanto que los productos agrícolas sólo fueron un componente más de una economía en la que plantas y animales silvestres eran explotados en forma intensiva. Con esta información la economía tardía se define como mixta, basada en la combinación de prácticas agrícolas con aportes significativos de caza y recolección, aun cuando la falta de datos adecuados impide medir la incidencia relativa de cada actividad (Medina 2008; Pastor 2007a). Dentro de este modelo, el maíz y la cerámica fueron extensamente utilizados, pero dentro de una estrategia que incluía cierto grado de movilidad a lo largo del ciclo anual.

¿Por qué las poblaciones tardías no desarrollaron una economía plenamente agrícola y la cazarecolección continuó siendo una actividad importante? Tucker (2006) sostiene que las poblaciones humanas tienden a devaluar subjetivamente las opciones económicas que implican períodos de espera, como la agricultura, independientemente de su rendimiento neto y productividad. Esta tendencia se acentúa cuando su producción se encuentra sujeta a niveles impredecibles de pérdida, como es el caso de las Sierras de Córdoba (Berberián y Roldán 2001; Medina y Pastor 2006; Piana de Cuestas 1992). En cambio, optan por actividades cuyos resultados son inmediatos y de menor costo de fallo, como la caza-recolección (Tucker 2006).

La incertidumbre respecto al resultado final de las cosechas pudo ser un factor que limitara una mayor dependencia hacia los productos agrícolas. Los grupos tardíos, en este sentido, optaron por continuar implementando prácticas predatorias mientras fuera posible. Las oportunidades para el aprovechamiento de recursos animales y vegetales silvestres fueron variables a lo largo del ciclo anual, siendo la primavera, estación en la que debía realizarse la siembra, el momento con menores posibilidades para la caza-recolección. De este modo, el aporte de los cultígenos no habría depen- 
dido de su productividad, sino de la ausencia de otras alternativas al inicio de la estación productiva (Medina 2008).

Las evidencias contempladas en este trabajo sostienen estos argumentos. Se defiende que aún cuando en el Periodo Prehispánico Tardío se practicó una agricultura de temporal basada en la tétrada americana (maíz, calabaza, alubia y quinua), ésta no habría implicado una alta inversión de tiempo y energía, imponiendo la necesidad de cierta movilidad para contrarrestar el agotamiento de los suelos y el crecimiento de malezas. Dado que la agricultura implicaba una cierta inversión de trabajo con resultados impredecibles, la subsistencia se habría complementado con aportes significativos de caza-recolección, que incluso pudieron constituir el total de la dieta en años poco favorables para la producción agrícola. Respecto a esto último, el padre Barzana en 1594 escribió

...tambien se sustentan de grandísima suma de algarroba, la cual cogen por los campos todos los años al tiempo que madura y hace della grandes depósitos; y cuando no llueve para coger maíz o el río no sale de madre para poder regar la tierra, pasan sus necesidades con esta algarroba... (citado por Berberián 1987: 254).

Los asentamientos residenciales a cielo abierto, en consecuencia, habrían sido ocupados en forma discontinua, reflejando la concentración estival de un conjunto de unidades domésticas para socializar, realizar actividades agrícolas, recolectar y capturar pequeños animales, así como para procesar, almacenar y consumir sus productos. También serían el locus donde se manufacturaban numerosos artefactos que luego serían utilizados a lo largo del año, sobre todo de aquellos que demandaban cierta estabilidad residencial y/o un combustible adecuado para su elaboración, como los recipientes cerámicos (Medina 2008, 2010).

Una vez finalizadas las tareas agrícolas y de recolección, el grupo co-residente se dispersaría hacia distintos puntos del paisaje para realizar actividades de caza-recolección y mantener la fluidez sociopolítica de la cual dependían para su reproducción social. De este modo, los grupos tardíos deberían ser clasificados como "agricultores móviles" (sensu Graham 1994: 107) que hicieron un uso estacional y diversificado del paisaje, donde las unidades domésticas o familiares constituyeron pequeños núcleos relativamente autónomos de producción, consumo y ocupación del espacio. Esto no excluiría la existencia de fuertes lazos comunitarios y una alta dinámica social reforzadas por la co-residencia estacional (Medina 2008), actividades de caza-recolección de carácter extradoméstico (Medina et al. 2011; Pastor 2007b), así como por códigos iconográficos compartidos en la decoración cerámica y el arte rupestre que se reproducirían cotidianamente con el uso de los recipientes o la ocupación de determinados paisajes (Medina 2010; Recalde y Pastor 2012).

\section{Conclusión y direcciones futuras}

La visión aquí defendida acerca de la economía y la movilidad tardía difiere de la tradicionalmente sostenida y otorga un gran dinamismo al proceso sociocultural prehispánico. Entre sus principales consecuencias, la nueva perspectiva entiende que el estudio del Período Prehispánico Tardío debe abandonar el concepto de sociedades "agrícolas" o "sedentarias", así como todas sus acepciones, ya que su uso ha llevado a enmascarar una gran variabilidad de formas de organización prehispánicas (Medina 2008). También pone en duda los indicadores de sedentarismo propuestos por Rafferty (1985: 128-137), de amplio consenso entre los arqueólogos, planteando la necesidad de ajustar la validez de algunos de elementos que integran la lista, como la presencia de viviendas, agricultura y tecnología cerámica ${ }^{3}$.

$\mathrm{La}$ incorporación de prácticas agrícolas $c a$. $1100 \mathrm{AP}$, de acuerdo con lo observado, no produjo cambios drásticos ni revolucionarios en las estrategias de asentamiento y subsistencia, en tanto que la caza-recolección continuó cubriendo una parte importante de la dieta. La agricultura, por el contrario, fue estacionalmente interrumpida cuando los recursos silvestres de mayor rendimiento se encontraban disponibles en otros puntos del paisaje.

Estos condicionantes llevan a concluir que las poblaciones tardías de las Sierras de Córdoba desarrollaron patrones de subsistencia y movilidad sumamente flexibles, donde se alternaron las estrategias de acuerdo con la disponibilidad estacional de recursos silvestres y las condiciones variables del entorno social. De este modo, el desarrollo de una economía diversificada se acompañaba de una alta movilidad residencial, momentos de dispersión/agregación de los grupos co-residentes y procesos estacionales de abandono de las prácticas productivas para abastecerse de recursos de caza y recolección.

El modelo aquí presentado está basado en las múltiples evidencias que actualmente se encuentran disponibles para el sector serrano de Córdoba. El mismo constituye un ejemplo arqueológico donde la incorporación de cultivos dio lugar a un patrón de subsistencia flexible sin derivar en una economía plenamente agrícola, con evidente potencial comparativo para el estudio de la transición 
hacia la producción de alimentos en otras regiones del mundo (Arnold 1999; Chilton 1999; Diehl 2005; Hard y Merrill 1992; Iriarte 2007; Madsen y Simms 1998; Pearsall 2004; Skibo y Blinman 1999; Smith 2001; Winterhalder y Kennett 2006). Sin embargo, el modelo no debe ser considerado inmutable ni definitivo. Seguramente, será redefinido con la continuidad de las investigaciones y la incorporación de nuevas herramientas teóricometodológicas que permitan analizar aspectos escasamente contemplados o condicionados por la falta de información.

En este sentido, será necesario ampliar las excavaciones en sitios residenciales a cielo abierto, con el propósito de dar cuenta del complejo mosaico de prácticas de subsistencia y movilidad de las sociedades tardías. La utilización de un marco teórico que tenga en cuenta las múltiples combinaciones entre agricultura, caza-recolección, sedentarismo y movilidad residencial, junto con metodologías de excavación sensibles a la discusión de estos aspectos y tendientes a la identificación en planta de agujeros de poste, pisos de ocupación, rasgos de vivienda, fogones, etc., serán pasos fundamentales dentro de esta perspectiva. En este sentido, las excavaciones de área abierta como las realizadas en Potrero de Garay (Berberián 1984) deben ser retomadas junto con la consideración de nuevos indicadores de movilidad residencial (i.e. densidad de agujeros de poste, características de los fogones, materiales utilizados, evidencias de remodelado, señales de abandono, superposición de pisos, etc.; Diehl 1997) y de producción agrícola (i.e. rasgos, funcionalidad de posibles artefactos agrícolas, microfósiles, macro-restos, etc.; Pearsall 2004). Al mismo tiempo, se debe profundizar en el conocimiento de la ocupación de los ambientes serranos de menor jerarquía agrícola, así como en los diversos patrones locales de articulación con los asentamientos residenciales (Pastor y Medina 2005; Pastor et al. 2012; Recalde 2008-09).

En forma paralela, se necesita un mayor número de dataciones directas por AMS sobre cultígenos. La mayoría de los sitios arqueológicos que presentan macro-restos o micro-fósiles de cultígenos fueron datados indirectamente mediante carbón. Es importante no dejar dudas de la asociación del cultígeno en sí mismo con la datación obtenida y establecer, por ejemplo, el rango temporal en el que cada taxón se introdujo dentro de la economía de las sociedades tardías.

El maíz y las alubias no deben recibir una atención desproporcionada por su mayor visibilidad arqueológica. Calabazas, quenopodáceas, tubérculos andinos (Solanum spp., Oxalis tuberosa) y de las tierras bajas (Ipomea batata, Canna edulis) fueron equivalentes al maíz en términos de kilocalorías y no tendrían que considerarse recursos secundarios en el contexto de una dieta diversificada, estacionalmente flexible y adversa al riesgo (Iriarte 2007).

Además, se deben ampliar los estudios de isotopos estables sobre restos humanos, una de las técnicas más efectivas para evaluar el impacto de la agricultura en la dieta de las poblaciones prehistóricas y sus cambios a través del tiempo (Barberena 2013). Esto se acentúa en el caso del maíz, una planta no local con un patrón fotosintético diferente y relativamente fácil de identificar respecto al de otras plantas nativas. Algunos avances ya fueron realizados mediante el estudio de colecciones depositadas en museos (Laguens et al. 2009), pero es necesario incrementar las muestras y establecer la ecología isotópica regional para establecer parámetros de comparación confiables (Barberena 2013). Asimismo, los estudios osteológicos pueden colaborar en la misma discusión. Los trabajos de Bordach et al. (1991) y González y Fabra (2011) marcaron el camino que debe ser ampliado.

Los grupos que ocuparon campamentos-base estacionales crearon hábitats continuamente perturbados por la actividad antrópica que favorecieron a ciertas plantas silvestres con alto valor nutricional, como Chenopodium spp. y Amaranthus spp. (Smith 1992). Gránulos de almidón de Chenopodium sp. fueron identificados adheridos a manos de moler en el sitio Quebrada del Real 1 (Pampa de Achala, Córdoba), lo cual indica la manipulación y explotación de estas plantas por lo menos desde fines del Holoceno Medio (Rivero y López 2011). La identificación en C.Pun.39 de Chenopodiaceae-Amaranthaceae a partir de polen en sedimentos datados en $c a$. $500 \mathrm{AP}$ y gránulos de almidón adheridos a fragmentos cerámicos, daría cuenta de la continuidad de estas prácticas, un aspecto que debe ser profundizado (Medina et al. 2008). En este sentido, el uso de la asociación cultivo/maleza no debe ser descartada en futuros estudios del Periodo Prehispánico Tardío, dada la resistencia de Chenopodiaceae-Amaranthaceae a las frecuentes sequías y heladas.

En la misma línea, las investigaciones arqueológicas necesitan considerar indicadores paleoambientales que permitan discutir las estrategias de movilidad y subsistencia a partir de su impacto en la vegetación a escala local y regional. Medina et al. (2008) realizaron una primera aproximación a esta problemática, discutiendo patrones de abandono de sitios residenciales tardíos y/o presencia de cultivos a partir de espectros polínicos. Sin embargo, las investigaciones requieren ser profundizadas con la incorporación de una perspectiva off- 
site (Pearsall 2004) sobre columnas de sedimentos de lagunas y pantanos para completar la discontinuidad de la información recuperada en los sitios arqueológicos. El estudio de las concentraciones de partículas de carbón, cambios en las frecuencias taxonómicas de polen y fitolitos de malezas y/o la ocurrencia de plantas domésticas en sí mismas son líneas de investigación fundamentales para avanzar en esta perspectiva. Los trabajos de Piovano et al. (2009) sobre núcleos de sedimentos de $c a$. 13.000 AP de la laguna de Mar Chiquita (Córdoba, Argentina) abren la puerta a esta posibilidad.

\section{Agradecimientos}

Este artículo es el resultado de investigaciones que han recibido subsidios del Consejo Nacional de Investigaciones Científicas y Técnicas (CONICET PIP 112-200801-02678) y de la Agencia Nacional de Promoción Científica y Tecnológica (PICT-2012-0995 y PICT-2012-1614). Deseamos expresar nuestro agradecimiento a Elizabeth Chilton, Michael Diehl, Gustavo Politis, Luis Borrero, Laura López y Julián Salazar, quienes aportaron bibliografía y comentarios que ayudaron a mejorar la calidad del manuscrito.

\section{Notas}

1. El término "sedentario" se utiliza aquí para indicar la ocupación anual de un asentamiento por lo menos por parte de la población (Rafferty 1985) y "residencialmente móviles" para señalar el cambio de residencia durante el año (Binford 1980; Diehl 1992). El número de veces que todo un grupo de co-residentes cambia la localización de su asentamiento es tomado como una medida de "movilidad residencial" (Diehl 1997).

2. La intensidad de la ocupación se refiere al rango de actividades desarrolladas en el sitio y el número de veces en que éstas ocurren. La duración de la ocupación es el tiempo en que el sitio fue utilizado, mientras que la continuidad es el grado en que la ocupación fue sostenida a lo largo del tiempo (Diehl 2005).

3. El problema no es que los atributos no se correlacionan con el sedentarismo, sino cómo la información fue utilizada en el marco de las investigaciones arqueológicas. En este sentido, indican circunstancias favorables para el sedentarismo más que una consecuencia del mismo (Diehl 2005).

\section{REFERENCIAS BIBLIOGRÁFICAS}

Aparicio, F. (1939): La Antigua Provincia de los Comechingones. Historia de la Nación Argentina I, Tiempos Prehistóricos y Protohistóricos (Academia Nacional de la Historia, ed.), Librería y Editorial El Ateneo, Buenos Aires: 359-386.

Arnold, P. (1999): Tecomates, residential mobility and Early Formative occupation in Coastal Lowland Mesoamérica. Pottery and People. A Dynamic Interaction (J. Skibo, G. Feinman, eds.), The University of Utah Press, Salt Lake City: 159-170.

BArberenA, R. (2013): Isotopic Studies of Foragers' Diet: Environmental Archaeological Approaches. Encyclopedia of Global Archaeology (C. Smith, ed.), Springer Science y Business Media, New York.

Berberián, E. (1984): Potrero de Garay: Una entidad sociocultural tardía de la región serrana de la Provincia de Córdoba (Rep. Argentina). Comechingonia, 4: 71-138.

Berberián, E. (1987): Crónicas del Tucumán. Siglo XVI. Editorial Comechingonia, Córdoba.

Berberián, E. (1999): Sierras Centrales. Nueva Historia de la Nación Argentina (Academia Nacional de Historia, ed.), Editorial Planeta, Buenos Aires: Tomo I: 135-158.

Berberián, E.; Bixio, B. (1987): La crónica de Gerónimo de Bibar y los aborígenes de la Provincia de Córdoba (República Argentina). Revista Española de Antropología Americana, 17: 197-234.

Berberián, E.; Roldán, F. (2001): Arqueología de las Sierras Centrales. Historia Argentina Prehispánica (E. Berberián, A. Nielsen, eds.), Editorial Brujas, Córdoba: 635-691.

Berberián, E.; RoldÁn, F. (2003): Limitaciones a la producción agrícola, estrategias de manejo de terrenos cultivables y ampliación de la dieta en comunidades formativas de la región serrana de la provincia de Córdoba. Relaciones de la Sociedad Argentina de Antropología, 28: 117-131.

Behrensmeyer, A. (1978): Taphonomic and ecologic information from bone weathering. Paleobiology, 4: 150162. 
BINFORD, L. (1980): Willow smoke and dog's tail: hunter-gatherer settlement systems and archaeological site formation. American Antiquity, 45: 4-20.

Bixio, B., Berberián, E. (1984): Etnohistoria de la región de Potrero de Garay (Pcia. de Córdoba - Rep. Argentina). Comechingonia, 3: 11-46.

Bordach, M.; Mendonça, O.; Español, V. (1991): Características antropo-físicas de los antiguos habitantes del Embalse de Río Tercero (Pcia. de Córdoba). Comechingonia, 7: 49-61.

Canals Frau, S. (1953): Las Poblaciones Indígenas de la Argentina. Editorial Sudamericana, Buenos Aires.

Chilton, E. (1999): Mobile farmers of pre-Contact southern New England: the archaeological and ethnohistoric evidence. Current Northeast Paleoethnobotany (J. Hart, ed.), New York State Museum Bulletin 949, Albany: 157-176.

Dantas, M.; Figueroa, G. (2008): Análisis Tecnológico y Funcional del Registro Cerámico del Valle de Salsacate y Pampas de Altura Adyacentes (Provincia de Córdoba, República Argentina). BAR International Series 1869, Oxford.

DieHL, M. (1992): Architecture as a material correlate of mobility strategies: some implications for the archaeological interpretation. Behavior Science Research, 26: 1-36.

DieHL, M. (1997): Changes in architecture and land use strategies in the American Southwest: Upland Mogollon Pithouse Dwellers. A.C. 200-1000. Journal of Field Archaeology, 24: 179-194.

DieHL, M. (2005): When corn was not the King. Subsistence and Resource Use Strategies of Early Agricultural Communities in Southern Arizona (M. Diehl, ed.), Anthropological Papers, 34, Center for Desert Archaeology, Tucson: 1-18.

Eerkens, J. (2003): Residential mobility and pottery use in the Western Great Basin. Current Anthropology, 44: 728-738.

Gifford, D.; IsaAC, G.; Nelson, C. (1980): Evidence for predation and pastoralism at Prolongated Drift: a pastoral Neolithic site in Kenya. Azania, 15:57-108.

GonzÁlez, A. (1943): Arqueología del Yacimiento Indígena de Villa Rumipal (Pcia. de Córdoba). Publicaciones del Instituto de Arqueología, Lingüística y Folklore "Dr. Pablo Cabrera" IV, Universidad Nacional de Córdoba, Córdoba.

GonzÁlez, C.; FABra, M. (2011): Estimaciones acerca de la salud de poblaciones que ocuparon las Sierras Centrales y Planicies Orientales (Córdoba, Argentina) en el Holoceno Tardío: una aproximación desde la antropología dental. Revista del Museo de Antropología, 4: 161-178.

Graham, M. (1994): Mobile Farmers. An Ethnoarchaeological Approach to Settlement Organization Among the Rarámuri of Northwestern Mexico. Internacional Monographs in Prehistory, Michigan.

Hard, R.; Merrill, W. (1992): Mobile agriculturalist and the emergence of sedentism: perspectives from Northern Mexico. American Anthropologist, 94: 601-620.

IRIARTE, J. (2007): New perpectives on plant domestication and the development of agriculture in the New World. Rethinking Agriculture. Archaeological and Ethnoarchaeological Perspectives (T. Denham, J. Iriarte, L. Vrydaghs, eds), Left Coast Press, Walnut Creek: 167-188.

Kunn, S. (1995): Mousterian Lithic Technology. An Ecological Perspective. Princeton University Press, Princeton.

Laguens, A. (1999): Arqueología del Contacto Hispano-Indígena. Un Estudio de Cambios y Continuidades en las Sierras Centrales de Argentina. BAR International Series 801, Oxford.

Laguens, A.; Bonnín, M. (2009): Sociedades Indígenas de las Sierras Centrales. Arqueología de Córdoba y San Luis. Editorial de la Universidad Nacional de Córdoba, Córdoba.

Laguens, A.; Fabra, M.; Santos, G.; Demarchi, D. (2009): Paleodietary inferences base on isotopic data for Pre-Hispanic population of the Central Mountain of Argentina. International Journal of Osteoarchaeology, 19: 237-249.

Layton, R.; Foley, R.; Williams, E. (1991): The transition between hunting and gathering and the specialized husbandry of resources. Current Anthropology, 32: 255-274.

López, L. (2005): Los pobladores productores de alimentos de las sierras de Córdoba. Primeras evidencias arqueobotánicas de los sitios Tala Cañada 1 y C.Pun.39. La Zaranda de Ideas, 1: 89-91.

Madsen, D.; Simms, S. (1998): The Fremont Complex: A behavioral perspective. Journal of World Prehistory, 12: 255-336.

Martín de Zurita, J. (1983): Etnohistoria del Departamento Pocho (Pcia. de Córdoba - Rep. Argentina) durante 
el siglo XVI. Comechingonia, 1: 113-149.

Medina, M. (2008): Diversificación Económica y Uso del Espacio en el Tardio Prehispánico del Norte del Valle de Punilla, Pampa de Olaen y Llanura Noroccidental (Córdoba, Argentina). Tesis Doctoral inédita. Facultad de Filosofía y Letras, Universidad de Buenos Aires, Buenos Aires.

Medina, M. (2010): Tecnología cerámica, subsistencia y uso del Espacio en el tardío prehispánico de las Sierras de Córdoba (Argentina). Revista Werkén, 13: 305-322.

Medina, M.; Grill, S.; LóPez, L. (2008): Palinología arqueológica: su implicancia en el estudio del prehispánico tardío de las Sierras de Córdoba (Argentina). Intersecciones en Antropología, 9: 99-112.

Medina, M.; López, L. (2007): Arqueobotánica del Sitio Puesto La Esquina 1 (Pampa de Olaen, Córdoba): Resultados Preliminares. Actas del XVI Congreso Nacional de Arqueología Argentina, Tras las Huellas de la Materialidad, Tomo III, San Salvador de Jujuy: 477-482.

Medina, M.; López, L.; Berberián, E. (2009): Agricultura y recolección en el Tardío Prehispánico de las Sierras de Córdoba (Argentina): el registro arqueobotánico de C.Pun.39. Arqueología, 15: 217-230.

Medina, M.; Pastor, S. (2006): Chacras dispersas. Una aproximación etnográfica y arqueológica al estudio de la agricultura prehispánica en la región serrana de Córdoba (Argentina). Comechingonia, 9: 103-121.

Medina, M.; Pastor, S. (2012): Zooarqueología de Sitios Residenciales Tardíos de las Sierras de Córdoba (Argentina, ca. 1100-300 AP): Avances y Perspectivas. Temas de Arqueología, Estudios Tafonómicos y Zooarqueológicos II (A. Acosta, D. Loponte, L. Mucciolo, eds), Instituto Nacional de Antropología y Pensamiento Latinoamericano, Buenos Aires: 45-66.

Medina, M.; Pastor, S.; Apolinaire, E.; Turnes, L. (2011): Late Holocene subsistence and social integration in Sierras of Córdoba (Argentina): the south-american ostrich eggshells evidence. Journal of Archaeological Science, 38: 2071-2078.

Muscio, H. (2004): Dinámica Poblacional y Evolución Durante el Período Agroalfarero Temprano en el Valle de San Antonio de los Cobres, Puna de Salta, Argentina. Tesis Doctoral inédita. Facultad de Filosofía y Letras, Universidad de Buenos Aires, Buenos Aires.

Nelson, M. (1991): The study of technological organization. Advances in Archaeological Method and Theory, 3: $57-100$.

Outes, F. (1911): Los tiempos prehistóricos y protohistóricos en la provincia de Córdoba. Revista del Museo de La Plata, Tomo VII (Segunda serie, Tomo IV): 261-374.

Pastor, S. (2005): El sitio Río Yuspe 14 (Pampa de Achala, Córdoba). Perspectivas sobre el uso prehispánico tardío de los ambientes serranos de altura. Mundo de Antes, 4: 87-104.

Pastor, S. (2007a): Arqueología del Valle de Salsacate y Pampas de Altura Adyacentes (Sierras Centrales de Argentina). Una Aproximación a los Procesos Sociales del Periodo Prehispánico Tardio (900-1573 d.C.). Tesis Doctoral inédita. Facultad de Ciencias Naturales y Museo, Universidad Nacional de La Plata, La Plata.

PAstor, S. (2007b): "Juntas y cazaderos". Las actividades grupales y la reproducción de las sociedades prehispánicas de las Sierras Centrales de Argentina. Procesos Sociales Prehispánicos en el Sur Andino. La Vivienda, la Comunidad y el Territorio (A. Nielsen, M. Rivolta, V. Seldes, M. Vázquez, P. Mercolli, eds.), Editorial Brujas, Córdoba: 361-376.

Pastor, S. (2010): Aproximación inicial a la arqueología del norte de la sierra de Guasapampa y cordón de Serrezuela (Córdoba, Argentina). Arqueología, 16: 151-174.

Pastor, S.; Berberián, E. (2007): Arqueología del sector central de las Sierras de Córdoba (Argentina). Hacia una definición de los procesos sociales del período prehispánico tardío (900-1573 d.C.). Intersecciones en Antropología, 8: 31-47.

PAstor, S.; López, L. (2010): Consideraciones sobre la agricultura prehispánica en el sector central de las Sierras de Córdoba. Arqueología de la Agricultura: Casos de Estudio en la Región Andina Argentina (A. Korstanje, M. Quesada, eds.), Editorial Magma, Tucumán: 208-233.

Pastor, S.; Medina, M. (2005): El Uso Prehispánico Tardío de los Ambientes Serranos de Altura. Investigaciones Arqueológicas en Pampa de Achala, de San Luis y de Olaen (Córdoba, Argentina). La Zaranda de Ideas, 1: 43-58.

Pastor, S; Medina, M.; Berberián, E. (2013): Poblados, Casas y Maizales. Arqueología de los Sitios Residenciales Tardíos de las Sierras de Córdoba (ca.1100-300 AP), Argentina Central. Revista Española de Antropología Americana, 43: 31-55.

Pastor, S.; Medina, M.; Recalde, A.; López, L.; Berberián, E. (2012): Arqueología de la Región Montañosa 
Central de Argentina. Avances en el Conocimiento de la Historia Prehispánica Tardía. Relaciones de la Sociedad Argentina de Antropología; 37: 89-112.

Pearsall, D. (2004): Plants and People in Ancient Ecuador: The Ethnobotany of the Jama River Valley. Case Studies in Archaeology, Jeffrey Quilter Series Editor, Belmont.

Piana de Cuestas, J. (1992): Los Indigenas de Córdoba Bajo el Régimen Colonial (1570-1620). Dirección General de Publicaciones de la Universidad Nacional de Córdoba, Córdoba.

Piovano, E.; Ariztegui, D.; Córdoba, F.; Cioccale, M.; Sylvestre, F. (2009): Hydrological Variability in South America below the Tropic of Capricorn (Pampas and Eastern Patagonia, Argentina) During the Last 13.0 ka. Past Climate Variability in South America and Surrounding Region (F. Vimeux, F. Sylvestre, M. Khodri, eds.), Springer, Dordrecht: 323-351.

RAFFerTy, J. (1985): The archaeological record on sedentariness: recognition, development and implication. Advances in Archaeological Method and Theory, 8: 113-156.

RECALDE, A. (2008-09): Movilidad estacional y representaciones rupestres. Primeras evidencias de ocupaciones estivales vinculadas con la explotación de ambientes chaqueños en las Sierras de Córdoba. Anales de Arqueología y Etnología, 63-64: 57-80.

Recalde, A.; Pastor, S. (2012): Contextos "públicos" y "privados" para la ejecución del arte rupestre en el valle de Guasapampa (Córdoba, Argentina). Latin American Antiquity, 23(3): 327-345.

Rivero, D.; LóPez, L. (2011): Evidencias del procesamiento de recursos vegetales por cazadores-recolectores de las Sierras de Córdoba en el período ca. 7000-2900 AP. Resúmenes de las IX Jornadas de Investigadores en Arqueología y Etnohistoria del Centro-Oeste del país, Universidad Nacional de Río Cuarto, Río Cuarto: 49-50.

Skibo, J.; Blinman, E. (1999): Exploring the origins of pottery on the Colorado Plateu. Pottery and People. A Dynamic Interaction (J. Skibo, G. Feinman, eds.), The University of Utah Press, Salt Lake City: 171-183.

Serrano, A. (1945): Los Comechingones. Serie Aborígenes Argentinos I. Instituto de Arqueología, Lingüística y Folklore de la Universidad Nacional de Córdoba, Córdoba.

Sмith, B. (1992): Rivers of Change. Essays on Early Agriculture in Eastern North America. Smithsonian Institution Press, Washington.

Smith, B. (2001): Low-Level food production. Journal of Archaeological Research, 9: 1-43.

Stevenson, M. (1982): Toward an understanding of site abandonment behavior: evidence from historic mining camps in the Southwest Yukon. Journal of Anthropological Archaeology, 1: 237-265.

Tucker, B. (2006): A future discounting explanation for the persistence of a mixed foraging-horticulture strategy among the Mikea of Madagascar. Behavioral Ecology and the Transition to Agriculture (D. Kennett, B. Winterhalder, eds.), University of California Press, Berkeley: 22-40.

WARD, G.; Wilson, S. (1978): Procedures for comparing and combining radiocarbon age determinations. Archaeometry, 20: 19-31.

Winterhalder, B.; Kennett, D. (2006): Behavioral Ecology and the transition from hunting and gathering to agriculture. Behavioral Ecology and the Transition to Agriculture (D. Kennett, B. Winterhalder, eds.), University of California Press, Berkeley: 1-21. 